\title{
Engaging patients in decision-making and behavior change to promote prevention
}

\author{
Alex H. Krist*, Sebastian T. Tong, Rebecca A. Aycock and Daniel R. Longo \\ Department of Family Medicine and Population Health, Virginia Commonwealth University, \\ Richmond, VA, USA
}

\begin{abstract}
Effectively engaging patients in their care is essential to improve health outcomes, improve satisfaction with the care experience, reduce costs, and even benefit the clinician experience. This article will address the topic of patient engagement directly and review the relationships between health literacy and patient engagement. While there are many ways to define patient and family engagement, this article will consider engagement as "patients, families, their representatives, and health professionals working in active partnership at various levels across the health care system - direct care, organizational design and governance, and policy making - to improve health and health care [Health Aff (Millwood) 32 (2013), 223-231]." We will specifically focus on the patient engagement and health literacy needs for three scenarios (1) decision-making, (2) health behavior change, and (3) chronic disease management; we will include the theoretical underpinnings of engagement, the systems required to better support patient engagement, how social determinants of health influence patient engagement, and practical examples to demonstrate approaches to better engage patients in their health and wellbeing. We will close by describing the future of patient engagement, which extends beyond the traditional domains of decision-making and self-care to describe how patient engagement can influence the design of the healthcare delivery system; local, state, and national health policies; and future research relevant to the needs and experiences of patients.
\end{abstract}

Keywords: Patient engagement, patient activation, patient education, health literacy, social determinants of health, delivery of healthcare

\section{Introduction}

This article addresses the topic of patient engagement directly and reviews the relationships between health literacy and patient engagement. While there are many ways to define patient and family engagement, this article considers engagement as "patients, families, their representatives, and health professionals working in active partnership at various levels across the health care system - direct care, organizational design and governance, and policy making - to improve health and health care." The authors specifically focus on the patient engagement and health literacy needs for three scenarios: (1) decisionmaking, (2) health behavior change, and (3) chronic disease management. The article addresses the theoretical underpinnings of engagement, the systems required to better support patient engagement, how social determinants of health influence patient engagement, and practical examples to demonstrate approaches to better engage patients in their health and wellbeing. The article closes by describing the future of patient engagement, which extends beyond the traditional domains of decision-making and self-care to describe how patient engagement can influence the design of the healthcare delivery system; local, state, and national health policies; and research relevant to the needs and experiences of patients.

\footnotetext{
${ }^{*}$ Corresponding author: Alex H. Krist, One Capital Square Room 631, 830 East Main Street, Richmond VA 23219. E-mail: alexander.krist@vcuhealth.org.
} 
The specific subtopics covered in the article are: the need for patient engagement; engaging patients to better understand decisions; engaging patients to improve health behaviors; engaging patients to improve chronic disease management; the influence of health's social determinants on engagement; health information seeking behaviors and engagement; as well as future directions.

\section{The need for patient engagement}

Several US studies recently reported coordinated care trials that actively engaged patients with chronic disease resulted in significant mortality reductions compared to a control group who only took appropriate medications $[18,19,55]$. The studies suggest chronically ill patient who are engaged in their care live longer than unengaged peers who otherwise receive similar treatment $[18,19,55]$. In other words, health and wellbeing are fostered by engaged and activated patients, who collaborate with their clinician to better manage care. In summarizing the hypothetical impact of widespread patient engagement on contemporary health care, Kish described the influence would be analogous to the introduction of a once-in-a-century blockbuster drug [38].

In addition, patient engagement demarcates an increasing shift from more paternalistic models of care in which clinicians tell patients what they should do (and often ineffectively), to one in which clinicians partner with patients. The collaborative partnership is intended to: help make better medical decisions; educate patients about how to stay healthy and manage conditions; develop systems and supports to activate patients; and sustain patient interest in their ongoing care.

The US Institute of Medicine's landmark report, Crossing the Quality Chasm, emphasized healthcare providers should be "respectful of and responsive to individual patient preferences, needs, and values," and ensure "patient values guide all clinical decisions [14]." In the US, the idea of engaging patients also has been advanced by the research funded by the Patient Centered Outcomes Research Institute (PCORI) and it is at the heart of national healthcare initiatives, such as Meaningful Use and the Medicare sharedsavings program for Accountable Care Organizations [20,67].

So why is patient engagement important in health care? What additional evidence is there to support the need to engage patients? What is the effect of varied health literacy on effective engagement? To begin, the need for patient engagement is posited as foundational because most adults spend little time in health care facilities and frequently are on their own to make appropriate, daily health decisions. This means patients need to be in control and the drivers of their health. Patient engagement further has an (a) ethical basis - engagement supports patient autonomy and self-determination, (b) interpersonal basis - engagement promotes confidence and trust in the clinician-patient relationship, and (c) educational basis - engagement improves knowledge, sets reasonable expectations, and reduces decisional conflict.

There is a practical need for engaging patients in their care as well. Many medical decisions have a trade-off of benefits and harms and sometimes there is a close balance of benefit to harm. Only by including patient values and preferences can a good decision be made [72]. Chronic disease management and health behavior change both must be done by the patient. Without complete buy-in and understanding of care and needed changes, a patient will not be able to effectively manage their health. Ultimately, the patient must suffer or enjoy the outcomes associated with any medical decision, test, treatment, or health behavior change.

In an international study of patients with "complex health needs" spanning 11 industrialized countries and focusing on the relationship between engagement and health care quality, substantial differences in the level of patient engagement between countries was identified. Consistently, countries with higher 
levels of engagement had better quality of care, lower medical error rates, and greater satisfaction in the experience of care [64]. Four case studies in diverse countries and health care settings further show the importance of engaging patients and the resulting improvements in health care quality and outcomes [44]. Collectively these findings demonstrate how patient engagement shifts the clinical paradigm from "what is the matter?" to more meaningfully discovering "what matters to you?"

There is a growing literature on how patient engagement impacts the experience and delivery of care. Minority patients frequently receive lower rates of preventive services. They suffer delays in diagnosis of diseases such as cancer, and once identified they even suffer delays in treatment. In a recent study, Sheppard has found that medical mistrust may contribute to these problems, something that could be overcome through effective patient engagement [71]. Survey data collected by Arora from cancer survivors demonstrates that better engagement increases the perception of personal control, increases trust, and decreases uncertainty [4]. Torres demonstrated that clinician communication styles are critically important to effective patient engagement and "good" communication creates a sense of not being rushed, a feeling like the clinician understands the patient, and a partnership built on trust [86]. Effective communication to better engage a diverse spectrum of patients with varying levels health literacy needs to be learned by all clinicians.

A review of proven strategies to enhance patient engagement identified three focus areas for engagement: improving health literacy, helping patients make appropriate health decisions, and improving the quality of care processes [16]. The Health Literate Care Model is an important tool to inform how attention to health literacy can improve patient engagement [39]. This model encourages clinicians to approach "all patients with the assumption that they are at risk of not understanding their health conditions or how to deal with them, and then subsequently confirming and ensuring patients' understanding." Across the spectrum of healthcare delivery, full engagement of the patient requires the patient to be able to obtain, process and communicate health information. Strategies to ensure that engagement activities are appropriate for a patient's health literacy can include adapting and simplifying language to decrease the risk of misunderstanding, providing examples that are relevant to the individual's lifestyle and cultural context, using visual representations of data, and integrating decision aids into care [22]. In a health literate care model, information needs to be presented in a manner that is congruent with a patient's ability to understand the material and span the domains in which health care occurs - the clinical setting, home, and community.

\section{Engaging patients TO better understand decisions}

\subsection{The evolution of patient engagement for decision-making}

Engaging patients in health care decision-making has significant benefits. Patients who participate in their decisions report higher levels of satisfaction with their care; have increased knowledge about conditions, tests, and treatment; have more realistic expectations about benefits and harms; are more likely to adhere to screening, diagnostic, or treatment plans; have reduced decisional conflict and anxiety; are less likely to receive tests or procedures which may be unnecessary; and, in some cases, even have improved health outcomes $[60,61,77]$.

Engaging patients in decisions has its basic grounding in the Nuremburg code which originated, mandated, and defined informed consent as a requirement for involving participants in research. Informed consent is the concept that individuals must be aware and understand what will be asked of them if 
they choose to participate and the risks and benefits of participating in a study. The information must be presented in a way that facilitates complete understanding - irrespective of the person's health literacy. In the mid-1970s, informed consent was extended to clinical practice requiring clinicians to disclose the risks and benefits of a medical procedure and then obtain patient permission before the procedure rather than patients simply yielding to, or complying with proposed medical care [6]. While this represents an improvement in patient engagement, it was mainly applied to surgical procedures and most efforts focused on getting signed consent rather than ensuring patient involvement in decision-making or even ensuring full comprehension of the procedure and alternatives [2,3].

\subsection{Key components of shared and informed decision-making}

In the mid-1980's, informed consent evolved in to a more collaborative relationship between patients and clinicians, where both parties shared information and came to joint decisions. The closely related concepts of informed decision-making and shared decision-making emerged. Shared decision-making has been defined as, "an approach where clinicians and patients share the best available evidence when faced with the task of making decisions, and where patients are supported to consider options, to achieve informed preferences [21]." In this model, the clinician's role is to elicit the patient's understanding, values, or reasoning and serve as a partner in decision making. According to the US Preventive Services Task Force (USPSTF), shared decision-making within the patient-clinician partnership universally encompasses a process in which both the patient and clinician share information with each other, take steps to participate in the decision-making process, and agree on a course of action [72].

Braddock defined seven elements that informed decision-making: (1) discussion of the patients role in decision making, (2) discussion of the clinical issue, (3) discussion of alternatives, (4) discussion of the pros and cons of alternatives, (5) discussion of uncertainties, (6) assessment of patient understanding, and (7) exploration of patient preference [9]. Braddock acknowledged that medical decisions vary in complexity and these elements will be employed to varying degrees depending on how straight forward or complex the decision. Embedded in each element is a recognition that in order for a patient to fully engage in any discussion there is need for the patient to have some health literacy. Clinicians should approach decision steps with attention to the patient's literacy needs and assess the patient's knowledge and understanding throughout.

\subsection{Implementing patient engagement for decision-making}

While some medical decisions are straightforward with one clear "right" choice, most decisions have multiple options each with a different set of advantages and disadvantages for patients and clinicians to consider. For some decisions, it is important to incorporate clinical information such as individual patient risks, the specifics of the condition, comorbidities, and potential prognoses. While this may be done by clinicians without much patient engagement, often patients may be the only source that knows, or has at least experienced all their medical history. Patient engagement is critical to ensure that all the medical information is being incorporated into these decisions. For other decisions, it may be more important to include patient's values, preferences, likelihood for adherence, and life circumstances. This scenario clearly involves patient engagement as only patients know this information. Effective discussions include both clinicians sharing clinical information about the options and patients sharing information about themselves.

Common examples of medical decisions include whether and how to make a health behavior changes, when to start and how to get preventive screening, management for acute or chronic conditions, how 
to prioritize competing health needs, and even when to change or stop a treatment. Some decisions are routine and occur frequently in practice such as when to start screening for breast cancer or how to be tested for colorectal cancer $[7,63,75,76]$. In one US primary care setting, nearly one in five patients seen for an office visit faced a routine decision about preventive care [43]. Other more major decisions, such as how to treat localized breast cancer or manage an abdominal aortic aneurysm, may only occur once in a patient's lifetime.

Traditionally, clinicians engage patients in decision-making during in-person visits. This may work well for major decisions, which occur infrequently, have obvious consequences, and may be amenable to clinicians and patients meeting on several occasions to make the decision. More routine decisions that are part of an office visit during which multiple issues are discussed are often overlooked by patients and clinicians. When asked, more than two thirds of patients report that they would like to share decisions with their clinician - routine and major. Sadly, this happens less than half the time $[93,96]$; conversations between clinicians and patients rarely include all elements of a good decision $[48,56,96]$; and while patients consider themselves knowledgeable about decisions, patients frequently have a poor understanding of the medical facts and often over-estimate the value of medical care [32].

One solution is to use decision aids and supports to help patients make medical decisions. These tools can ensure patients receive information in a standardized format that includes all critical content, presents information in a culturally appropriate manner, and uses language and images to ensure understanding across a range of health literacy needs. Decision aids are not routinely used in clinical care $[31,35,45,47$, 62]. Key barriers include time, expense, perceived legitimacy, capacity, ability to integrate into workflow, lack of clinician training and comfort with decision aids, and an environment that has not made routine use a cultural norm [73]. Despite these barriers many good decision aids have been developed. A host of organizations have cataloged and made available a range of high quality decision aids tailored to a range of literacy levels and cultural norms as well as trainings and resources to help clinicians better implement shared decision-making (see Table 1).

Krist proposes that to be effective, decision aids must also be integrated into the clinical workflow realistically, patients undergo a "decision journey [43]." This journey requires support over time, al-

Table 1

Organizations and resources that promote and support informed and shared decision-making for patients and clinicians

\begin{tabular}{ll}
\hline Supports & Organization \\
\hline Decision aid standards & - International Patient Decision Aids Standards (IPADS) \\
Library of decision aids & - Agency for Healthcare Research and Quality - Effective Healthcare Program - Decision Aids \\
and supports & - Healthwise (also available at WebMD by typing "decision" followed by health topic) \\
& - Mayo Clinic Shared Decision-Making National Resource Center \\
& - National Health Service - Decision Aids \\
& - Ottawa Hospital Decision Center \\
Medical decision-making & - American Academy on Communication Healthcare \\
societies & - Informed Medical Decisions Foundation \\
& - Society for Medical Decision Making \\
Tools to promote and & - Agency for Healthcare Research and Quality - Healthcare Innovation Exchange \\
implement shared & - Dartmouth Center for Shared-Decision Making \\
decision-making in practice & - Institute for Healthcare Improvement \\
Video decision aids & - Emmi \\
& - Foundation for Informed Decision-Making \\
& - Health Dialog \\
\hline
\end{tabular}




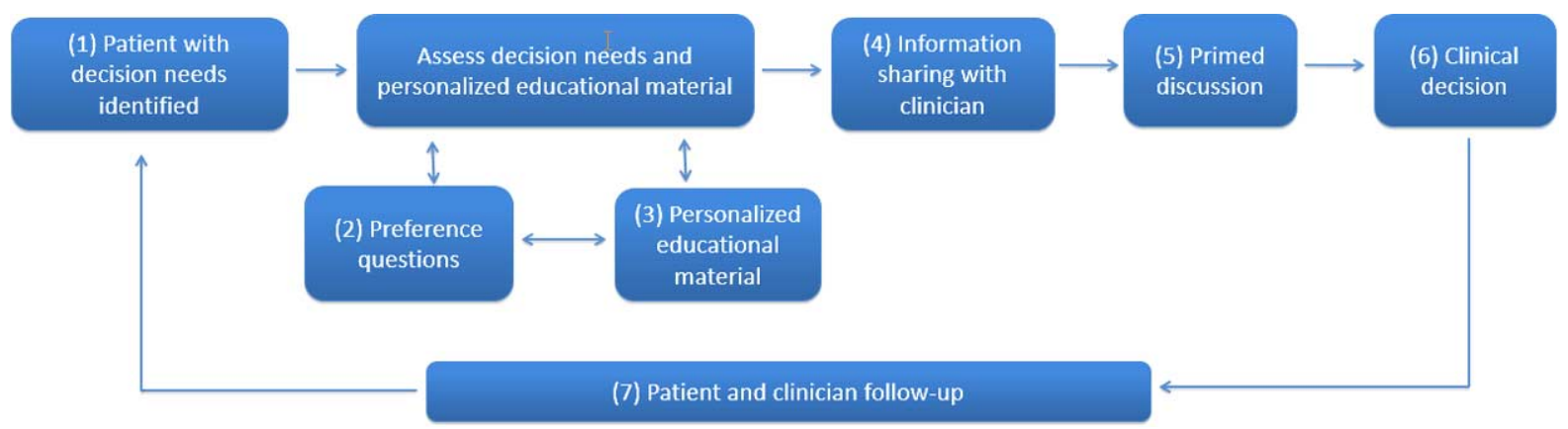

Fig. 1. A workflow to better engage patients throughout their decision-making journeys. To better engage patients in their decisions, this workflow, which several practices programmed into their patient portal and electronic health record, guides patients and clinicians through a series of seven steps: (1) based on electronic health record data, patients with decision needs are identified, and the patient portal reaches contacts patients outside the confines of an office visit to start considering decision options; (2) the patient portal walks patients through an intake that assesses personal preferences, knowledge, needs, and readiness to make a decision; (3) the portal provides personalized educational material tailored to the patient's stated preferences and decision stage; (4) the portal allows the patient to share their preferences and decision needs with their clinician; (5) the clinician reviews the information prior to a visit, priming the discussion so the clinician is aware of the patient's needs; (6) the patient and clinicians are able to make a more informed and shared decision; and (7) the electronic health record and patient portal can follow-up with both the clinician and patient to make sure the decision is acted upon consistent with the patient's wishes (modified from [43]).

lowing patients to contemplate options, gather additional information, confer with family and friends, consider individual preferences, and address their personal worries or concerns. Clinicians can serve as trusted advisors during this decision journey. One example of systematically supporting decision journeys is how a group of practices used their patient portal to promote cancer screening decisions (Fig. 1). The system anticipated the patients' decision needs; delivered decision support prior to visits; allowed patients to tailor decision supports to their interests and needs; collected patient-reported information about where they were with their decision journey, what they wanted to discuss with their clinician, and their fears; shared the patient reported information with their clinician; set a decision-making agenda; and even provided follow-up on next steps [43]. Routine implementation of similar workflows and processes, whether technology-based or not, has great potential to improve care, address health literacy issues, and better engage patients in decision-making.

\section{Engaging patients to improve health behaviors}

Similar to increasing patients' participation in medical decision making, clinicians need to engage patients to effect desired health behavior changes. Research has shown that incorporating patient's goals and motivations into planned behavior change increases the likelihood that a patient will be successful with behavior change. Multiple strategies have been developed to increase patient involvement in health behavior change, including the 5 As, the 5 Rs, and Motivational Interviewing. Similar to shared decision-making, each of these strategies require the clinician to elicit the patient's reasons for change and incorporate those reasons into the behavior change plan. Through this process the clinician can also ascertain a patient's understanding of their health care and address any misconceptions.

The 5 As is a framework that can help guide clinician actions to better engage patients who are working towards health behavior change [1,27,92]. The major steps to the five As include: (1) Ask every patient about health behaviors, (2) Advise patients with an unhealthy behavior in a clear, strong, and 
personalized manner to modify the behavior, (3) Assess the patient's willingness to change the health behavior (sometimes referred to instead as seeking Agreement on the patient's willingness to change the health behavior), (4) Assist the patient in modifying the health behavior, and (5) Arrange for follow-up. For many behaviors, A1 through A3 can occur during one encounter and may take only a few moments. Conversely, A4 and A5 - assisting patients and arranging follow-up - often require intensive support extended over a period of time. For example, interventions to help patients eat right, exercise, or lose weight often take dozens of hours of face to face contact over a period of months from multiple members of a multidisciplinary team $[88,89]$. The exception to the intensive time and resource requirement for A4 and A5 is counseling patients to quit smoking and counseling patients against risky drinking behaviors (not treating alcoholism). A4 and A5 can be done effectively in a matter of minutes during one encounter with brief follow-up and support. While more intensive interventions to help patients quit smoking and limit risky drinking are more likely to result in lasting heath behavior changes, brief interventions for these two behaviors do have some efficacy [23,59].

For patients that are not ready to make a health behavior change, the five Rs is a tool that clinicians can use to help patients move to a stage of readiness to change their health behavior. The 5 Rs prompts the clinician to: (1) discuss the Relevance of the change for the patient (e.g. smoking may be contributing to your getting so many colds and missing work so often), (2) discuss the Risks of continuing the unhealthy behavior, (3) discuss the Rewards of adopting a healthy behavior, (4) identify Roadblocks to changing the behavior, and (5) Repetition of the personalized five Rs message at each visit $[25,26]$. The last $R$, Repetition, stresses the importance of reiterating the 5 Rs to help motivate patients to change behaviors whenever possible, so that when the patient is ready to make changes, the assistance and support is available.

Motivational Interviewing is a third strategy that leverages a patients' values and goals to initiate and maintain behavior change. Motivational Interviewing is defined as "a client-centered, directive method for enhancing intrinsic motivation to change by exploring and resolving ambivalence [57]." One of the key components of this definition is a patient's ability to develop intrinsic motivation which requires that he/she has a knowledge of how the behavior change directly relates to personal goals. In order to enhance intrinsic motivation, the patient must be able to relate the behavior change to their sense of self, their self in the context of family and community, and their other values and roles. Effective patient education and support tailored to the patient's needs and health literacy can increase their sense of self-efficacy. This education can help patients learn how diseases progresses and how changing behaviors can make a clear impact on their health. Making the behavior change relevant to the patient's experience may alleviate shame and guilt and instill hope that change is possible.

Sadly, unhealthy behaviors account for nearly $40 \%$ of premature deaths and substantial morbidity in the US [58]. Engaging patients in health behavior change has been clearly shown to improve health and patients commonly report a clinician's advice to change an unhealthy behavior as a key motivating factor for change [28,79]. Yet few patients report being asked regularly about their health behaviors; only 10-20\% of smokers report being told to quit smoking by their clinician; less than $20 \%$ of obese patients report being told by their clinician that they are overweight; and only $2-5 \%$ of patients in need of intensive diet, exercise, and weight loss counseling actually receive assistance [46,69].

There are many reasons why health behavior counseling is done poorly in practice including lack of time, competing demands, inadequate resources and support, limited training in health behavior counseling, and even lack of confidence in effecting change in patients [12,34,78]. Exceptional practices and health systems are increasingly trying to better address health behavior counseling by building the infrastructure support necessary for intensive assistance and follow-up, creating multidisciplinary teams 
that can address the range of patient needs, and having dedicated staff to follow-up and provide ongoing assistance and motivation $[8,68]$. To be successful all care team members must have defined roles, be effective patient communicators, understand the patient's information and social needs, and pay attention to each individual's health literacy. New payment models that reward improved outcomes and value-based care may further support and enhance these practice efforts. Alternatively, practices can form partnerships with existing community programs designed to help patients improve health behaviors. These clinical community linkages can often more effectively address patient's needs by building on the strengths of each partner - clinicians to Ask, Advise, and Assess patient's readiness to change and community programs to provide the intensive Assistance and follow-up in the places that patients live, work, and play $[41,42,94]$. One framework, proposed by Krist, depicts how clinical practices and community programs can work together to better engage patients in health behavior change and care in general (Fig. 2).

\section{Engaging patients for chronic disease care}

Understanding how patients can be engaged in self-management of chronic conditions is also important given today's growing prevalence of chronic disease. For example, an estimated 70 million Americans live with hypertension and 29 million live with type 2 diabetes mellitus. Another significant proportion of the US population have the precursors to these chronic diseases - 70 million have prehypertension and 86 million have prediabetes. The number and prevalence of chronic conditions that patients must live with continues to expand.

One commonly promoted model for designing systems to better address chronic conditions is the Chronic Care Model (CCM). Developed in the mid-1990s by Wagner, the CCM identifies key health system elements needed to provide effective chronic disease management and prevention [90]. These elements include (a) the community, (b) the health system, (c) self-management support, (d) delivery system design, (e) decision support, and (f) clinical information systems. When these elements function synergistically they result in a more informed, engaged, and activated patient as well as a more prepared, proactive practice team. The patient and the practice team can have more productive interactions, clinicians can better assist patients, and patients can better manage their health - all leading to improved outcomes. Use of the CCM to inform and guide the care delivery system has been evaluated extensively and is demonstrated to both improve health outcomes and cost-effectiveness [13].

When designed effectively, the healthcare and community delivery systems can provide the tools to help patients to become more informed, engaged and activated in their care. Engaged patients are more likely to practice healthy behaviors, eating right, exercising, and not smoking, mitigating any harms from their chronic condition. They seek and use more health information from a wide range of sources to learn about their condition and ways to manage it. And they better self-manage their condition by following up with their primary care clinician and specialists, getting needed tests to monitor their condition, adhering to daily medications, and participating in self-monitoring activities. Effective healthcare and community delivery systems should encourage and support these activities in a manner attentive to the patient's health literacy and health information needs.

\section{The influence of social determinants on engagement and literacy}

There is growing attention to the relationship between social determinants and whether clinicians can effectively engage patients in their care. It has long been known that health outcomes are affected by the 


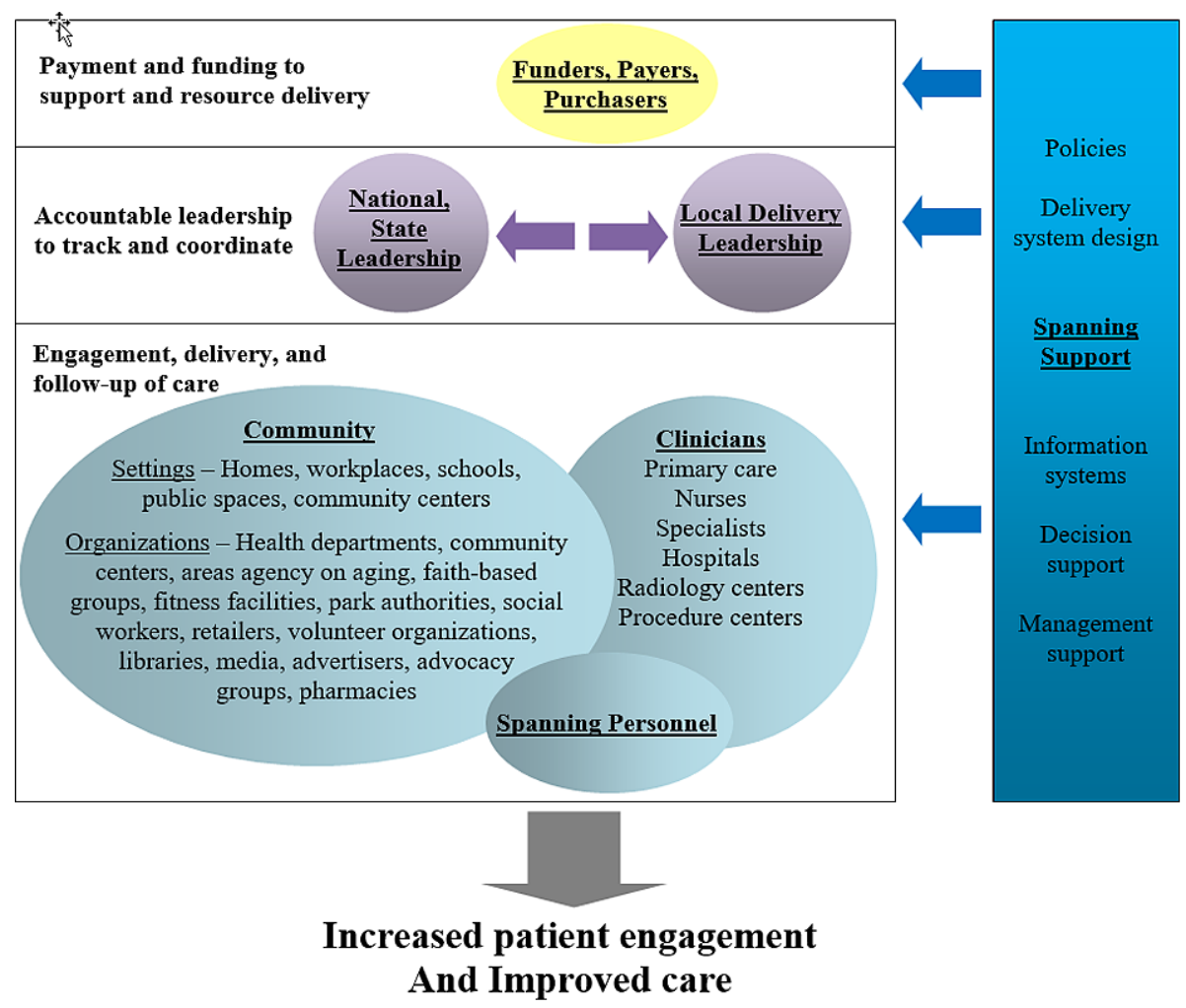

Fig. 2. A Framework for How Clinical Practices and Community Programs Can Partner to Better Engage Patients in Care. A framework depicting how funders, policy makers, communities, and clinicians can work together with the support of personnel and infrastructure to link the care delivery systems. Funders, payers, and purchasers are tasked with financing the infrastructure needed to support integrating the clinical and community care systems. National and state leadership are empowered with the authority, resources, and responsibility to foster integrations across regions. Local leaders are the regional organizations that step forward to oversee and support local tailoring and integration activities. Community is the setting where individuals live work, and play and where the stakeholders who serve them are located. Community organizations are care providers that deliver the community elements of a clinical-community integration. Clinicians are care providers that deliver the clinical elements of a clinical-community integration. Spanning personnel are staff who specialize in helping people traverse the clinical and community settings to obtain care. Spanning support (which includes policies, delivery system design, information systems, decision support, and management support) are essential ingredients to support integrations at all levels depicted in the framework (modified from [41]).

social determinants of health, including socioeconomic status, education, ethnicity, race, and community of residence. The Institute of Medicine first drew attention to this problem, with a focus on racial and ethnic disparities, in their 2003 report, Unequal Treatment: Confronting Racial and Ethnic Disparities in Health Care [81]. The report highlighted that health outcome disparities could not be explained by merely lack of access to care, such as insurance status or availability of care. In addition, inherent stereotyping of patients and biases of clinicians contributed to poorer quality of care for minority patients. While concordance between clinician and patient in ethnicity, race, and gender have been shown to be important contributors [82], patient engagement barriers extend beyond these factors. Several studies have reported that clinicians are verbally dominant and engage less in patient centered communication in encounters with ethnic minority patients $[15,36]$. This lack of engagement with patients is even worse when a language barrier exists between the clinician and patient. 
Clinicians are often also unable to fully comprehend the struggles with transportation, finances, housing and other economic barriers that patients of lower socioeconomic status may face. While patients with higher income may often live in the same communities as their clinicians, poorer patients either have to travel long distances to receive health care from clinics where they may be stereotyped or are served in clinics by clinicians who drive in from higher income communities. Unless directly asked, patients may often hesitate to bring up structural barriers to receipt of care and clinicians may not be aware of their barriers.

These barriers, whether created from ethnic differences, income disparities, geographic barriers, or inherent communication gaps, are often not addressed through intentional efforts to better engage patients in their care processes. In fact, special efforts need to be made since those who face barriers from social determinants have traditionally been disenfranchised within the health care system. A recent report published by the National Academies of Science, A Framework for Educating Health Professionals to Address the Social Determinants of Health, discusses processes in which some of these issues may be addressed. The framework includes three pillars: education, community and organization.

In terms of education, increased efforts to train clinicians in cultural competency may improve patient engagement. Defined as "a set of congruent behaviors, attitudes and policies that come together in a system, agency or among professionals and enable that system, agency or those professions to work effectively in cross-cultural situations," cultural competency may be one way clinicians can improve communication with patients and better facilitate patient engagement in care processes [17]. In 2012, the Association of American Medical Colleges and the Association of Schools of Public Health released a joint report suggesting core competencies in cultural competency for health professional students. Such curricular elements may include (1) improvement in culturally appropriate communication behavior, (2) situational awareness, (3) adaptability and (4) knowledge of core cultural issues [84].

In terms of community, joint efforts by clinicians and health systems to be involved in the communities that patients live may help facilitate better patient engagement. Through such efforts, community resources can be leveraged that may reduce social barriers that patients may face to accessing health care in a timely and appropriate fashion. Interian suggests that joint efforts by community organizations and health care organizations may help better educate patients, improve the way that structural barriers such as problems with transportation, finances and housing are addressed, and improve communication between patients and clinicians [33].

Organizational efforts may also help with patient engagement. Kauffman calls for more research is needed to address health disparities and studies need to include "hard to reach patients [37]." When including patients in outreach such as developing patient advisory councils or seeking community input, health care organizations may benefit from intentionally recruiting and including their more disenfranchised patients. From a long-term perspective, the work that health professional training schools are doing to increase minority enrolment and recruit culturally and economically diverse students may also help with future efforts to better engage patients.

While much work still needs to be done to eliminate the disparities in patient engagement, recent efforts have at least drawn attention to this issue. Attention has helped develop frameworks to understand disparities and create curricula for health professional students to learn about and start proactively addressing such disparities. 


\section{Patients health information seeking behaviors}

Information is central to a patient being engaged in their decisions, care, and self-management. With the advent of the internet, mobile technologies, and increasingly powerful search engines, patients can now instantaneously access all kinds of information anywhere they like to help guide their health with the touch of a button. Some patients still rely solely on the receipt of health information from clinicians, yet many more use a combination of approaches. Receiving information from a trusted clinician can be good - it can prevent a patient from being misled by inaccurate or commercially biased information. However, not actively seeking health information can be a missed opportunity. Many local and national organizations are working to raise awareness on the power of health information by promoting the need to get informed, directing patients to health information, and even creating information, ranging from educational material about health to reports on the quality of care from hospitals and clinicians to interactive and personalized tools to manage daily activities.

There are several models that explain how, why, and where patients seek health information. One model advanced in by Longo (Fig. 3) [49,51,52], identifies two axes of information seeking behaviors: active-passive and aware-unaware. Not only do patients fall into a spectrum of preferring to be active or passive seekers of health information, but patients fall into a spectrum of knowing that they need health information or knowing that health information is available. Further, there is a range of settings and sources that patients use information. Even patients involved in active searches for information are informed by passive information that they come across during usual activities of living. These passive sources include newspaper articles, television talk shows, billboards, and magazines. Passive sources can be useful or potentially misleading, particularly if commercially biased. Naturally, information needs and activities vary over time based on a multitude of patient and contextual factors. Some of these factors are modifiable and can be improved, resulting in increased health information seeking and improved health literacy. Although further research is clearly needed to identify best strategies for engaging patients in information seeking.

The information that patients can access has been continually and rapidly evolving, including the format of information, content and focus of information, and even where information is accessed. In the 1980s, a wealth of consumer information provided by professional organizations and advocacy groups emerged. Patients could access this information in lay press articles, brochures from their clinician, directly from the producer, and in community settings. This same information quickly moved to the internet and new innovative information sources emerged such as patient provided information. With the internet, individuals can reach wide audiences to share their experiences, create virtual support groups, and learn from others experiencing similar conditions; this is exemplified by tools such as patients likeme [65].

Now mobile devices, wearables, and the hyperconnectivity of personal health information is creating a new era of mobile health. Clinicians and health care systems are designing patient portals that can be accessed on the web or any mobile device $[40,66,74]$. Through integration of clinical information and patient reported information, these systems can anticipate patient needs and decisions, personalize educational content to better speak to the patient, reach out to patients outside of clinical encounters, and transform care from being reactive to proactive $[43,95]$. Asynchronous communication with clinicians and virtual visits can further facilitate information exchange between patients and clinicians. However, the impact of these clinician-provided information sources have on health outcomes is largely unknown and much research is needed about how these new approaches can better engage and activate patients in their care [29]. 
Variables Influencing Patient/Consumer Information-Seeking Behaviors Behavior and Information Use

Contextual

Health status, health care structure, delivery of care, information environment, information seeking for self, family member, or friend at risk or with current medical problem, interoersonal social supports, networks

\section{Personal}

Demographic factors, socioeconomic factors, health history, genetics, stress, education, culture, language, attitudes, behaviors, current health status, cognitive ability, interpersonal communication motives

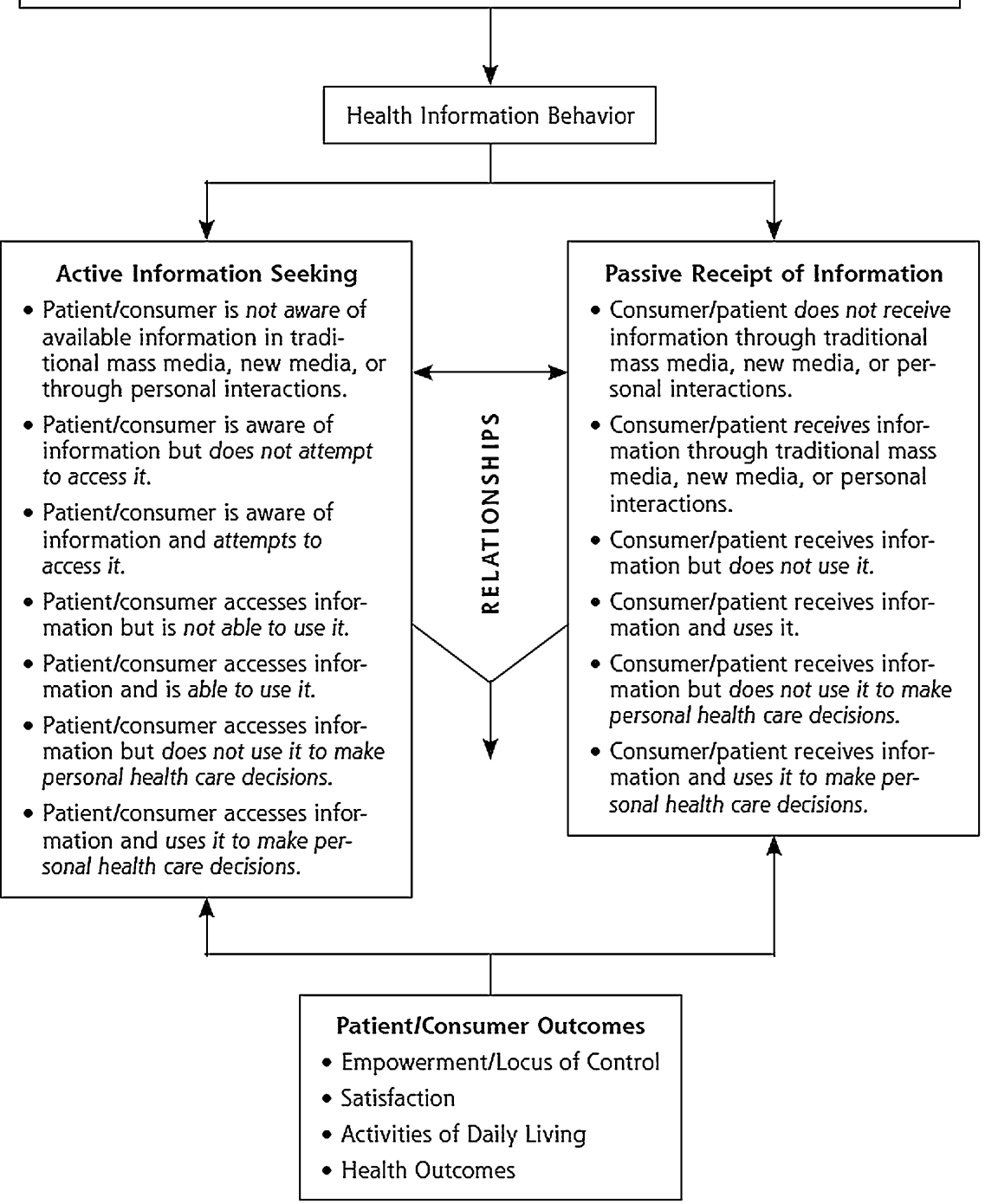

Fig. 3. A Model to Describe Patient's Health Information Seeking Behaviors (Reproduced with permission from Health Information Seeking, Receipt, and Use in Diabetes Self-Management, July/August, 2010, Vol 8, No 4, issue of Annals of Family Medicine Copyright@2010 American Academy of Family Physicians. All Rights Reserved.) 
Similarly, smart devices have become nearly ubiquitous, with an estimated 3.5 devices per every human on earth in 2015 [85]. These devices have resulted in an explosion of applications ("apps") that can receive, collect, tailor, and transmit health information, allowing patients to self-digitize for health often referred to now as Mobile Health or mHealth. mHealth has the potential to scale the delivery of highly personalized information directly to users and share the information with the user's clinician to better support care [80]. To date the development and dissemination of these resources has largely been driven by industry and consumer demand. There are few active, completed, or published studies that rigorously test $m$ Health interventions in randomized controlled trials to evaluate the impact on health outcomes [83], and there is less information focusing on how mHealth impacts patient engagement or understanding of health information [5]. In a recent systematic review, Free and colleagues only identified 42 well designed trials in North American, Europe, Asia, and Australia that showed improvements in patient self-management of diseases, clinician communication, and appointment attendance [24]. Given the potential uses of $m$ Health, much more research is needed.

Despite the proliferation of health information there is a paucity of data about the type of health information vulnerable and less health literate consumers want, particularly as it relates to newer technologies. Several studies have engaged disadvantaged populations to identify what health information would most help them with their care for a range of health topics [50,70]. Generally, two sets of desired information are commonly identified - a need for information to assist them in selecting clinicians and basic health information to assist them in self-management. Regardless of race and ethnicity, patients express a greater need for self-management information than other forms of information. Most importantly patients expressed a need for basic, straightforward information. There is some evidence for higher uptake of mobile internet technology by minorities and disadvantaged populations $[30,54,91]$. Accordingly, some disparities experts posit that mHealth tools have the potential to narrow disparities [87]. Yet for the most part, clinicians at safety-net practices report minimal use of even basic mobile technology in their practices [10].

Efforts are needed to both encourage and motivate patients to seek health information as well as to create high quality, patient-centered health information that is written at a basic reading level, free of jargon, culturally sensitive, non-biased, and available in multiple languages. Yet it is also important to understand where patients are with their information needs [53]. Some patients will have basic information needs, such as information about a condition, test, or treatment. Others may have more advanced needs such as information about nuanced differences between options or where to receive treatment. Some may just want information to understand and feel comfortable with care recommended by their clinician. Providing patients information mismatched to their needs is unlikely to be useful. A holistic approach is needed to providing patients information that takes into account the patient's diverse needs, desired sources, and various situations.

\section{Future directions}

There is clear evidence that working to respectfully encourage patient engagement improves health outcomes and wellbeing for patients. As discussed in this article, engagement is critical across the lifespan and across the disease spectrum, from prevention to chronic disease control. Recent decades have seen great advances in patient engagement for clinical decision-making, promoting healthy behaviors, and self-management of chronic conditions. Understanding and addressing health literacy is a central underpinning to all of these activities. 
Despite clear advances in our understanding of the value of patient engagement and effective strategies to engage patients in health, much more is needed. There are clear evidence gaps that deserve further work. Further research is needed to understand how to better inform and engage patients in decisions and self-management. Needs range from improving timely access to information for patients, ensuring that information is clear and understandable, ensuring that patients understand the importance of the information, and designing information to be actionable. As information becomes increasingly accessible from a host of venues, helping patients to navigate the morass of information and better integrating diverse sources of information could reduce patient confusion about what they need. A massive challenge will be to learn how to better redesign the healthcare delivery system to make evidence-based patient engagement part of routine care. This is especially challenging given how many decisions and health behaviors benefit from patient engagement. The health care system will also need to learn how to receive and interpret a growing expanse of patient reported information from wearables and other devices. This information may not only be able to help clinicians with decisions, but also be a mechanism in of itself to engage patients. Clinicians will also need to build and study partnerships with community resources that can extend engagement outside the clinical setting and better support patients in their daily lives. Given the diversity of patients and their needs, a host of solutions will be needed to engage patients and understanding how to match these solutions with individual patients will be critical. This challenge may be particularly important for more disadvantaged individuals and those with lower health literacy.

The future of patient engagement will need to continue to advance these activities, but also move beyond, to authentically engage patients not only in their health care, but in designing and implementing care delivery systems, developing local and national health policies, and directing health research [95]. Patient insights and lived experiences can ensure that healthcare systems and programs align with their priorities, address their needs, and are delivered in an accessible manner. Patients can help set healthcare systems' organizational priorities, participate in governance decisions, and define organizational strategies and activities. Similarly, patients can ensure that legislative, regulatory, and funding priorities reflect their needs. Patient engagement for research can help to frame research questions, select outcomes, develop study protocols, support recruitment, interpret results, translate research findings into lay language, disseminate results, and even sustain interventions.

Efforts will be needed to help patients realistically participate in these activities. Patient engagement will need to become the cultural norm. Patients will need training and support to meaningfully participate. Yet collectively, these higher levels of patient engagement can improve our very systems for promoting health.

\section{References}

[1] Agency for Healthcare Research and Quality, Five major steps, to intervention (The "5 A's"). 2012 [cited 2016 Oct]. Available from: http://www.ahrq.gov/professionals/clinicians-providers/guidelines-recommendations/tobacco/5steps.html.

[2] G.J. Annas, Informed consent, Annual Review of Medicine 29 (1978), 9-14, Epub 1978/01/01. doi:10.1146/annurev.me. 29.020178.000301.

[3] G.J. Annas and F.H. Miller, The empire of death: How culture and economics affect informed consent, in the US, the UK, and Japan, American Journal of Law \& Medicine 20(4) (1994), 357-394, Epub 1994/01/01.

[4] N.K. Arora, K.E. Weaver, M.L. Clayman, I. Oakley-Girvan and A.L. Potosky, Physicians' decision-making style and psychosocial outcomes among cancer survivors, Patient Educ Couns. 77(3) (2009), 404-412, Epub 2009/11/07. doi:10. 1016/j.pec.2009.10.004.

[5] S. Barello, S. Triberti, G. Graffigna, C. Libreri, S. Serino, J. Hibbard et al., EHealth for patient engagement: A systematic review, Frontiers in Psychology 6 (2015), 2013, Epub 2016/01/19. 
[6] T.L. Beauchamp and J.F. Childress, Principles of Biomedical Ethics, 7th edn, Oxford University Press, New York, 2013 , pp. xvi-459.

[7] K. Bibbins-Domingo, D.C. Grossman, S.J. Curry, K.W. Davidson, J.W. Epling Jr., F.A. Garcia et al., Screening for colorectal cancer: US Preventive Services Task Force recommendation statement, JAMA 315(23) (2016), 2564-2575, Epub 2016/06/16. doi:10.1001/jama.2016.5989.

[8] T. Bodenheimer, E.H. Wagner and K. Grumbach, Improving primary care for patients with chronic illness, JAMA 288(14) (2002), 1775-1779. doi:10.1001/jama.288.14.1775.

[9] C.H. Braddock 3rd, K.A. Edwards, N.M. Hasenberg, T.L. Laidley and W. Levinson, Informed decision making in outpatient practice: Time to get back to basics, JAMA 282(24) (1999), 2313-2320. doi:10.1001/jama.282.24.2313.

[10] A. Broderick and F. Haque, Mobile health and patient engagement in the safety net, Issue Brief (Commonw Fund) 9 (2015), 1-9, Epub 2015/06/05.

[11] K.L. Carman, P. Dardess, M. Maurer, S. Sofaer, K. Adams, C. Bechtel et al., Patient and family engagement: A framework for understanding the elements and developing interventions and policies, Health Aff (Millwood) 32(2) (2013), $223-231$. Epub 2013/02/06. doi:10.1377/hlthaff.2012.1133.

[12] R.M. Carpiano, S.A. Flocke, S.H. Frank and K.C. Stange, Tools, teamwork, and tenacity: An examination of family practice office system influences on preventive service delivery, Prev Med 36(2) (2003), 131-140. doi:10.1016/S00917435(02)00024-5.

[13] K. Coleman, B.T. Austin, C. Brach and E.H. Wagner, Evidence on the chronic care model in the new millennium, Health Affairs 28(1) (2009), 75-85. doi:10.1377/hlthaff.28.1.75.

[14] Committee on Quality of Health Care in America and Institute of Medicine Crossing the Quality Chasm: A New Health System for the 21st Century, National Academy Press, Washington, DC, 2001.

[15] L.A. Cooper, D.L. Roter, K.A. Carson, M.C. Beach, J.A. Sabin, A.G. Greenwald et al., The associations of clinicians' implicit attitudes about race with medical visit communication and patient ratings of interpersonal care, American Journal of Public Health 102(5) (2012), 979-987. doi:10.2105/AJPH.2011.300558.

[16] A. Coulter, Patient engagement - what works?, J Ambul Care Manage. 35(2) (2012), 80-89. doi:10.1097/JAC. 0b013e318249e0fd.

[17] T. Cross, B. Bazron, K. Dennis and M. Isaacs, Towards a Culturally Competent System of Care, Georgetown University Child Development Center, ed., CASSP Technical Assistance Center, Washington, DC, 1989.

[18] A. Darkins, S. Kendall, E. Edmonson, M. Young and P. Stressel, Reduced cost and mortality using home telehealth to promote self-management of complex chronic conditions: A retrospective matched cohort study of 4,999 veteran patients, Telemedicine Journal and e-Health: The Official Journal of the American Telemedicine Association. 21(1) (2015), 70-76. Epub 2014/05/21.

[19] A. Darkins, P. Ryan, R. Kobb, L. Foster, E. Edmonson, B. Wakefield et al., Care coordination/home telehealth: The systematic implementation of health informatics, home telehealth, and disease management to support the care of veteran patients with chronic conditions, Telemedicine Journal and e-Health: The Official Journal of the American Telemedicine Association. 14(10) (2008), 1118-1126, Epub 2009/01/06. doi:10.1089/tmj.2008.0021.

[20] Electronic health records and meaningful use. The Office of the National Coordinator for Health Information Technology, 2015 [cited 2016 Sept]. Available from: http://www.healthit.gov/providers-professionals/meaningful-usedefinition-objectives.

[21] G. Elwyn, D. Frosch, R. Thomson, N. Joseph-Williams, A. Lloyd, P. Kinnersley et al., Shared decision making: A model for clinical practice, J Gen Intern Med. 27(10) (2012), 1361-1367, Epub 2012/05/24. doi:10.1007/s11606-012-2077-6.

[22] G. Elwyn, S. Laitner, A. Coulter, E. Walker, P. Watson and R. Thomson, Implementing shared decision making in the NHS, BMJ 341 (2010), c5146. Epub 2010/10/16.

[23] M. Fiore, United States. Tobacco Use and Dependence Guideline Panel. Treating tobacco use and dependence: 2008 update. 2008 update ed. Rockville, MD: US Dept. of Health and Human Services, Public Health Service, 2008, pp. xvii256.

[24] C. Free, G. Phillips, L. Watson, L. Galli, L. Felix, P. Edwards et al., The effectiveness of mobile-health technologies to improve health care service delivery processes: A systematic review and meta-analysis, PLoS Medicine. 10(1) (2013), e1001363. Epub 2013/03/06.

[25] K. Glanz, B. Rimer and K. Viswanath, Health Behavior and Health Education, 4th edn, Wiley, New York, 2008.

[26] K. Glanz and B.K. Rimer, Theory at a Glance. A Guide for Health Promotion Practice. US Department of Health and Human Services, National Institute of Health, 2005 [cited 2009 May]; 2nd:[Available from: http://www.cancer.gov/PDF/ 481f5d53-63df-41bc-bfaf-5aa48ee1da4d/TAAG3.pdf.

[27] M.G. Goldstein, J. DePue, A. Kazura and R. Niaura, Models for provider-patient interaction: Applications to health behavior change, in: Handbook for Health Behavior Change, Spinger, New York, 1998, pp. 85-113.

[28] M.G. Goldstein, E.P. Whitlock and J. DePue, Multiple behavioral risk factor interventions in primary care. Summary of research evidence, Am J Prev Med. 27(Suppl. 2) (2004), 61-79. 
[29] C.L. Goldzweig, G. Orshansky, N.M. Paige, A.A. Towfigh, D.A. Haggstrom, I. Miake-Lye et al., Electronic patient portals: Evidence on health outcomes, satisfaction, efficiency, and attitudes: A systematic review, Ann Intern Med. 159(10) (2013), 677-687, Epub 2013/11/20. doi:10.7326/0003-4819-159-10-201311190-00006.

[30] B.W. Hesse, C. Hanna, H.A. Massett and N.K. Hesse, Outside the box: Will information technology be a viable intervention to improve the quality of cancer care?, Journal of the National Cancer Institute Monographs 2010(40) (2010), 81-89, Epub 2010/04/14. doi:10.1093/jncimonographs/lgq004.

[31] L. Hill, M.R. Mueller, S. Roussos, M. Hovell, J. Fontanesi, J. Hill et al., Opportunities for the use of decision aids in primary care, Fam Med 41(5) (2009), 350-355, Epub 2009/05/07.

[32] R.M. Hoffman, C.L. Lewis, M.P. Pignone, M.P. Couper, M.J. Barry, J.G. Elmore et al., Decision-making processes for breast, colorectal, and prostate cancer screening: The DECISIONS survey, Med Decis Making. 30(Suppl. 5) (2010), 53S64S. Epub 2010/10/15.

[33] A. Interian, R. Lewis-Fernández and L.B. Dixon, Improving treatment engagement of underserved US racial-ethnic groups: A review of recent interventions, Psychiatric Services 64(3) (2013), 212-222. doi:10.1176/appi.ps.201100136.

[34] C.R. Jaen, K.C. Stange and P.A. Nutting, Competing demands of primary care: A model for the delivery of clinical preventive services, J Fam Pract. 38(2) (1994), 166-171, Epub 1994/02/01.

[35] M. Jimbo, G.K. Rana, S. Hawley, M. Holmes-Rovner, K. Kelly-Blake, D.E. Nease Jr. et al., What is lacking in current decision aids on cancer screening?, CA Cancer J Clin. 63(3) (2013), 193-214, Epub 2013/03/19. doi:10.3322/caac.21180.

[36] R.L. Johnson, D. Roter, N.R. Powe and L.A. Cooper, Patient race/ethnicity and quality of patient-physician communication during medical visits, American Journal of Public Health 94(12) (2004), 2084-2090. doi:10.2105/AJPH.94.12. 2084.

[37] K.S. Kauffman, M. Ross, B. Barnet, E. Onukwugha and C.D. Mullins, Engaging Hard-to-Reach Patients in PatientCentered Outcomes Research, 2013.

[38] L. Kish, The blockbuster drug of the century: an engaged patient, 2012 [cited Sept 2016]. Available from: http:// healthstandards.com/blog/2012/08/28/drug-of-the-century/.

[39] H.K. Koh, C. Brach, L.M. Harris and M.L. Parchman, A proposed 'health literate care model' would constitute a systems approach to improving patients' engagement in care, Health Aff (Millwood) 32(2) (2013), 357-367. Epub 2013/02/06.

[40] A.H. Krist, E. Peele, S.H. Woolf, S.F. Rothemich, J.F. Loomis, D.R. Longo et al., Designing a patient-centered personal health record to promote preventive care, BMC Med Inform Decis Mak 11 (2011), 73, Epub 2011/11/26. doi:10.1186/ 1472-6947-11-73.

[41] A.H. Krist, D. Shenson, S.H. Woolf, C. Bradley, W.R. Liaw, S.F. Rothemich et al., Clinical and community delivery systems for preventive care: An integration framework, Am J Prev Med. 45(4) (2013), 508-516, Epub 2013/09/21. doi:10. 1016/j.amepre.2013.06.008.

[42] A.H. Krist, S.H. Woolf, C.O. Frazier, R.E. Johnson, S.F. Rothemich, D.B. Wilson et al., An electronic linkage system for health behavior counseling effect on delivery of the 5A's, Am J Prev Med. 35(Suppl. 5) (2008), S350-S358.

[43] A.H. Krist, S.W. Woolf, C. Hochheimer, R.T. Sabo, P. Kashiri, R.M. Jones et al., Harnessing information technology to inform patients facing routine decisions, Ann Fam Med. (2017), In press.

[44] J. Laurance, S. Henderson, P.J. Howitt, M. Matar, H. Al Kuwari, S. Edgman-Levitan et al., Patient engagement: Four case studies that highlight the potential for improved health outcomes and reduced costs, Health Aff (Millwood) 33(9) (2014), 1627-1634, Epub 2014/09/10. doi:10.1377/hlthaff.2014.0375.

[45] F. Legare, S. Ratte, D. Stacey, J. Kryworuchko, K. Gravel, I.D. Graham et al., Interventions for improving the adoption of shared decision making by healthcare professionals, Cochrane Database Syst Rev. 2010(5) (2010), CD006732. Epub 2010/05/14.

[46] R.R. Leverence, R.L. Williams, A. Sussman and B.F. Crabtree, Obesity counseling and guidelines in primary care: A qualitative study, Am J Prev Med. 32(4) (2007), 334-339, Epub 2007/03/27. doi:10.1016/j.amepre.2006.12.008.

[47] G.A. Lin, M. Halley, K.A. Rendle, C. Tietbohl, S.G. May, L. Trujillo et al., An effort to spread decision aids in five California primary care practices yielded low distribution, highlighting hurdles, Health Aff (Millwood) 32(2) (2013), 311320, Epub 2013/02/06. doi:10.1377/hlthaff.2012.1070.

[48] B.S. Ling, J.M. Trauth, M.J. Fine, M.K. Mor, A. Resnick, C.H. Braddock et al., Informed decision-making and colorectal cancer screening: Is it occurring in primary care?, Med Care. 46(9 Suppl. 1) (2008), S23-S29. Epub 2008/09/09.

[49] D.R. Longo, Understanding health information, communication, and information seeking of patients and consumers: A comprehensive and integrated model, Health Expect. 8(3) (2005), 189-194. doi:10.1111/j.1369-7625.2005.00339.x.

[50] D.R. Longo, B.F. Crabtree, M.B. Pellerano, J. Howard, B. Saver, E.L. Hannan et al., A qualitative study of vulnerable patient views of type 2 diabetes consumer reports, The Patient. 9(3) (2016), 231-240, Epub 2015/11/09.

[51] D.R. Longo, B. Ge, M.E. Radina, A. Greiner, C.D. Williams, G.S. Longo et al., Understanding breast cancer patient's perceptions: Health information-seeking behaviour and passive information receipt, J of Comm and Healthcare 2(2) (2009), 184-206. doi:10.1179/cih.2009.2.2.184.

[52] D.R. Longo, S.L. Schubert, B.A. Wright, J. LeMaster, C.D. Williams and J.N. Clore, Health information seeking, receipt, and use in diabetes self-management, Ann Fam Med. 8(4) (2010), 334-340, Epub 2010/07/21. doi:10.1370/afm.1115. 
[53] D.R. Longo and S.H. Woolf, Rethinking the information priorities of patients, JAMA 311(18) (2014), 1857-1858, Epub 2014/05/16. doi:10.1001/jama.2014.3038.

[54] M.L. Lustria, S.A. Smith and C.C. Hinnant, Exploring digital divides: An examination of eHealth technology use in health information seeking, communication and personal health information management in the USA, Health Informatics Journal 17(3) (2011), 224-243, Epub 2011/09/23.

[55] D. McCarthy, K. Mueller and J. Wren, The Commonwealth Fund Case Study. Kaiser Permanente: bridging the quality divide with integrated practices, group accountability, and health information technology, 2009 [cited 2016 Sept]. Available from: http://www.commonwealthfund.org/ /media/files/publications/case-study/2009/jun/1278_mccarthy_kaiser_ case_study_624_update.pdf.

[56] A. McQueen, L.K. Bartholomew, A.J. Greisinger, G.G. Medina, S.T. Hawley, P. Haidet et al., Behind closed doors: Physician-patient discussions about colorectal cancer screening, J Gen Intern Med. 24(11) (2009), 1228-1235, Epub 2009/09/19. doi:10.1007/s11606-009-1108-4.

[57] R.W. Miller and S. Rollnick, Motivational Interviewing: Preparing People to Change Addictive Behavior, The Guilford Press, New York, New York, 1991.

[58] A.H. Mokdad, J.S. Marks, D.F. Stroup and J.L. Gerberding, Actual causes of death in the United States, 2000, JAMA 291(10) (2004), 1238-1245.

[59] V.A. Moyer, Screening and behavioral counseling interventions in primary care to reduce alcohol misuse: US Preventive Services Task Force recommendation statement, Ann Intern Med. 159(3) (2013), 210-218, Epub 2013/05/24.

[60] A.M. O'Connor, C.L. Bennett, D. Stacey, M. Barry, N.F. Col, K.B. Eden et al., Decision aids for people facing health treatment or screening decisions, Cochrane Database Syst Rev. 2009(3) (2009), CD001431. Epub 2009/07/10.

[61] A.M. O'Connor, F. Legare and D. Stacey, Risk communication in practice: The contribution of decision aids, BMJ 327(7417) (2003), 736-740. doi:10.1136/bmj.327.7417.736.

[62] A.M. O'Connor, J.E. Wennberg, F. Legare, H.A. Llewellyn-Thomas, B.W. Moulton, K.R. Sepucha et al., Toward the 'tipping point': Decision aids and informed patient choice, Health Aff (Millwood) 26(3) (2007), 716-725, Epub 2007/05/09. doi:10.1377/hlthaff.26.3.716.

[63] K.C. Oeffinger, E.T. Fontham, R. Etzioni, A. Herzig, J.S. Michaelson, Y.C. Shih et al., Breast cancer screening for women at average risk: 2015 guideline update from the American cancer society, JAMA 314(15) (2015), 1599-1614, Epub 2015/10/27. doi:10.1001/jama.2015.12783.

[64] R. Osborn and D. Squires, International perspectives on patient engagement: Results from the 2011 Commonwealth Fund Survey, J Ambul Care Manage. 35(2) (2012), 118-128, Epub 2012/03/15. doi:10.1097/JAC.0b013e31824a579b.

[65] PatientsLikeMe. [cited 2017 Jan]. Available from: https://www.patientslikeme.com/.

[66] J.D. Ralston, K. Coleman, R.J. Reid, M.R. Handley and E.B. Larson, Patient experience should be part of meaningful-use criteria, Health Aff (Millwood) 29(4) (2010), 607-613. doi:10.1377/hlthaff.2010.0113.

[67] D.R. Rittenhouse, S.M. Shortell and E.S. Fisher, Primary care and accountable care-two essential elements of deliverysystem reform, N Engl J Med. 361(24) (2009), 2301-2303, Epub 2009/10/30. doi:10.1056/NEJMp0909327.

[68] M.C. Rosal, J.K. Ockene, R. Luckmann, J. Zapka, K.V. Goins, G. Saperia et al., Coronary heart disease multiple risk factor reduction. Providers' perspectives, Am J Prev Med 27(Suppl. 2) (2004), 54-60.

[69] S.F. Rothemich, S.H. Woolf, R.E. Johnson, A.E. Burgett, S.K. Flores, D.W. Marsland et al., Effect on cessation counseling of documenting smoking status as a routine vital sign: An ACORN study, Ann Fam Med. 6(1) (2008), 60-68. doi:10.1370/ afm.750.

[70] L.J. Rutten, N.K. Arora, A.D. Bakos, N. Aziz and J. Rowland, Information needs and sources of information among cancer patients: A systematic review of research (1980-2003), Patient Educ Couns. 57(3) (2005), 250-261, Epub 2005/05/17. doi:10.1016/j.pec.2004.06.006.

[71] V.B. Sheppard, D. Mays, T. LaVeist and K.P. Tercyak, Medical mistrust influences black women's level of engagement in BRCA 1/2 genetic counseling and testing, Journal of the National Medical Association 105(1) (2013), 17-22, Epub 2013/07/19. doi:10.1016/S0027-9684(15)30081-X.

[72] S.L. Sheridan, R.P. Harris and S.H. Woolf, Shared decision making about screening and chemoprevention. A suggested approach from the US Preventive Services Task Force, Am J Prev Med. 26(1) (2004), 56-66.

[73] C.G. Shultz and M. Jimbo, Decision aid use in primary care: An overview and theory-based framework, Fam Med 47(9) (2015), 679-692, Epub 2015/10/17.

[74] A.L. Silvestre, V.M. Sue and J.Y. Allen, If you build it, will they come? The kaiser permanente model of online health care, Health Aff (Millwood) 28(2) (2009), 334-344. doi:10.1377/hlthaff.28.2.334.

[75] A.L. Siu, Screening for breast cancer: US Preventive Services Task Force recommendation statement, Ann Intern Med. 164(4) (2016), 279-296, Epub 2016/01/13. doi:10.7326/M15-2886.

[76] A.L. Siu, K. Bibbins-Domingo, D.C. Grossman and M.L. LeFevre, Convergence and divergence around breast cancer screening, Ann Intern Med. 164(4) (2016), 301-302, Epub 2016/01/13. doi:10.7326/M15-3065.

[77] D. Stacey, F. Legare, N.F. Col, C.L. Bennett, M.J. Barry, K.B. Eden et al., Decision aids for people facing health treatment or screening decisions, Cochrane Database Syst Rev. 2014(1) (2014), CD001431. Epub 2014/01/29. 
[78] K.C. Stange, S.J. Zyzanski, C.R. Jaen, E.J. Callahan, R.B. Kelly, W.R. Gillanders et al., Illuminating the 'black box'. A description of 4454 patient visits to 138 family physicians, J Fam Pract. 46(5) (1998), 377-389.

[79] B. Starfield, L. Shi and J. Macinko, Contribution of primary care to health systems and health, Milbank Q. 83(3) (2005), 457-502.

[80] S.R. Steinhubl, E.D. Muse and E.J. Topol, Can mobile health technologies transform health care?, JAMA 310(22) (2013), 2395-2396, Epub 2013/10/26.

[81] Institute of Medicine, B.D. Smedley, A.Y. Stith and A.R. Nelson, eds, Unequal Treatment: Confronting Racial and Ethnic Disparities in Health Care, National Academies Press, Washington, DC, 2003.

[82] R.L. Street Jr., K.J. O’Malley, L.A. Cooper and P. Haidet, Understanding concordance in patient-physician relationships: Personal and ethnic dimensions of shared identity, Annals of Family Medicine 6(3) (2008), 198-205. doi:10.1370/afm. 821.

[83] E.F. Sutton and L.M. Redman, Smartphone applications to aid weight loss and management: Current perspectives, Diabetes, Metabolic Syndrome and Obesity: Targets and Therapy 9 (2016), 213-216, Epub 2016/08/04.

[84] C.R. Teal and R.L. Street, Critical elements of culturally competent communication in the medical encounter: A review and model, Social Science \& Medicine 68(3) (2009), 533-543.

[85] E.J. Topol, S.R. Steinhubl and A. Torkamani, Digital medical tools and sensors, JAMA 313 (2015), 353-354, Epub 2015/01/28.

[86] E. Torres, D.O. Erwin, M. Trevino and L. Jandorf, Understanding factors influencing Latina women's screening behavior: A qualitative approach, Health Educ Res 28(5) (2013), 772-783, Epub 2012/11/08. doi:10.1093/her/cys106.

[87] N. Turner-Lee, B.D. Smedley and J. Miller, Minorities, Mobile Broadband and the Management of Chronic Diseases, Joint Center for Political and Economic Studies, Washington DC, 2012.

[88] US Preventive Services Task Force, Screening for and management of obesity in adults, 2012 [cited 2013 Jan]. Available from: http://www.uspreventiveservicestaskforce.org/uspstf/uspsobes.htm.

[89] US Preventive Services Task Force, Screening for obesity in children and adolescents, 2010 [cited 2013 Mar]. Available from: http://www.uspreventiveservicestaskforce.org/uspstf/uspschobes.htm.

[90] E.H. Wagner, B.T. Austin, C. Davis, M. Hindmarsh, J. Schaefer and A. Bonomi, Improving chronic illness care: Translating evidence into action, Health Affairs 20(6) (2001), 64-78. doi:10.1377/hlthaff.20.6.64.

[91] D.S. Wakefield, R.L. Kruse, B.J. Wakefield, R.J. Koopman, L.E. Keplinger, S.M. Canfield et al., Consistency of patient preferences about a secure Internet-based patient communications portal: Contemplating, enrolling, and using, American Journal of Medical Quality: The Official Journal of the American College of Medical Quality 27(6) (2012), 494-502, Epub 2012/04/21.

[92] E.P. Whitlock, C.T. Orleans, N. Pender and J. Allan, Evaluating primary care behavioral counseling interventions: An evidence-based approach, Am J Prev Med. 22(4) (2002), 267-284. doi:10.1016/S0749-3797(02)00415-4.

[93] S.H. Woolf, A.H. Krist, R.E. Johnson and P.S. Stenborg, Unwanted control: How patients in the primary care setting decide about screening for prostate cancer, Patient Educ Couns. 56(1) (2005), 116-124. doi:10.1016/j.pec.2003.12.002.

[94] S.H. Woolf, A.H. Krist and S.F. Rothemich, Joining Hands: Partnerships Between Physicians and the Community in the Delivery of Preventive Care, Center for American Progress, Washington DC, 2006.

[95] S.H. Woolf, E. Zimmerman, A. Haley and A.H. Krist, Authentic engagement of patients and communities can transform research, practice, and policy, Health Aff (Millwood) 35(4) (2016), 590-594, Epub 2016/04/06. doi:10.1377/hlthaff.2015. 1512.

[96] T. Wunderlich, G. Cooper, G. Divine, S. Flocke, N. Oja-Tebbe, K. Stange et al., Inconsistencies in patient perceptions and observer ratings of shared decision making: The case of colorectal cancer screening, Patient Educ Couns. 80(3) (2010), 358-363, Epub 2010/07/30. doi:10.1016/j.pec.2010.06.034. 